\title{
Corrigendum: NovelTTC37 mutations in a patient with immunodeficiency without diarrhea: extending the phenotype of trichohepatoenteric syndrome
}

\author{
Nicholas L. Rider ${ }^{1}$, Bertrand Boisson ${ }^{2}$, Soma Jyonouchi ${ }^{3}$, Eric P. Hanson ${ }^{4}$, Sergio D. Rosenzweig ${ }^{5}$, \\ Jean-Laurent Casanova ${ }^{2,6,7}$ and Jordan S. Orange ${ }^{1 *}$ \\ 1 Department of Immunology, Allergy and Rheumatology, Baylor College of Medicine, Texas Children's Hospital, Houston, TX, USA \\ 2 St. Giles Laboratory of Human Genetics of Infectious Diseases, Rockefeller University, New York, NY, USA \\ ${ }^{3}$ Department of Allergy-Immunology, The Children's Hospital of Philadelphia, Philadelphia, PA, USA \\ ${ }^{4}$ Immunodeficiency and Inflammation Unit, National Institute of Arthritis, Musculoskeletal and Skin Disease, National Institutes of Health, Bethesda, MD, USA \\ ${ }^{5}$ Laboratory of Host Defense, National Institute of Allergy and Infectious Disease, National Institutes of Health, Bethesda, MD, USA \\ ${ }^{6}$ Necker Hospital for Sick Children, Imagine Institute, INSERM, HHMI, Paris Descartes University, Paris, France \\ 7 Howard Hughes Medical Institute, New York, NY, USA \\ ${ }^{*}$ Correspondence: ude.mcb@egnaro
}

Edited and reviewed by:

Pediatrics Editorial Office, Frontiers, Switzerland

Keywords: trichohepatoenteric syndrome, primary immunodeficiency, ectodermal dysplasia, trichorrhexis nodosa, antibody deficiency, chronic diarrhea

\section{A corrigendum on}

Novel TTC37 mutations in a patient with immunodeficiency without diarrhea: extending the phenotype of trichohepatoenteric syndrome

by Rider NL, Boisson B, Jyonouchi S, Hanson EP, Rosenzweig SD, Cassanova JL, et al. Front. Pediatr. (2015) 3:2. doi: 10.3389/fped.2015.00002

During the preparation of the paper the authors misspelled the name of author Jean-Laurent Casanova as Jean-Laurent Cassanova. The author list should read:
Nicholas L. Rider, Bertrand Boisson, Soma Jyonouchi, Eric P. Hanson, Sergio D. Rosenzweig, Jean-Laurent Casanova and Jordan S. Orange

The authors would like to apologize for any potential inconvenience caused.

The original article has been updated.

Conflict of Interest Statement: The authors declare that the research was conducted in the absence of any commercial or financial relationships that could be construed as a potential conflict of interest.

Received: 23 March 2015; accepted: 30 March 2015; published online: 16 April 2015.

Citation: Rider NL, Boisson B, Jyonouchi S, Hanson EP, Rosenzweig SD, Casanova J-L and Orange JS (2015)
Corrigendum: Novel TTC37 mutations in a patient with immunodeficiency without diarrhea: extending the phenotype of trichohepatoenteric syndrome. Front. Pediatr. 3:28. doi: 10.3389/fped.2015.00028

This article was submitted to Pediatric Hematology and Immunology, a section of the journal Frontiers in Pediatrics.

Copyright () 2015 Rider, Boisson, Jyonouchi, Hanson, Rosenzweig, Casanova and Orange. This is an openaccess article distributed under the terms of the Creative Commons Attribution License (CC BY). The use, distribution or reproduction in other forums is permitted, provided the original author(s) or licensor are credited and that the original publication in this journal is cited, in accordance with accepted academic practice. No use, distribution or reproduction is permitted which does not comply with these terms. 